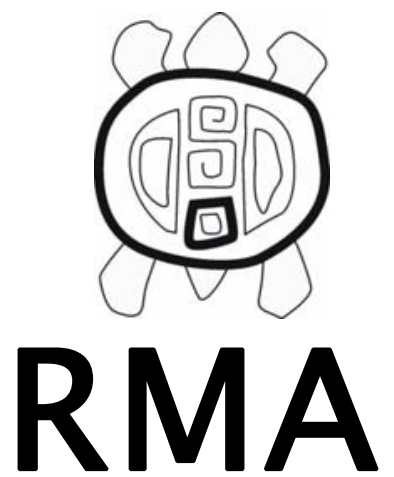

Museología

\title{
En la sombra de la arqueología argentina: Jorge von Hauenschild y la formación de la colección von Hauenschild del Museo de Antropología (Universidad Nacional de Córdoba)
}

Henrik B. Lindskoug

Becario FONCYT, Museo de Antropología Facultad de Filosofía y Humanidades, Universidad Nacional de Córdoba henrikblindskoug@gmail.com

\section{Resumen}

Se presenta un estudio acerca de la colección von Hauenschild, radicada actualmente en el Museo de Antropología, Facultad de Filosofía y Humanidades de la Universidad Nacional de Córdoba, Argentina. El trabajo explora la historia de la colección en el tiempo y en el espacio, así cómo su formación por parte del ingeniero alemán Jorge von Hauenschild, en la provincia de Santiago del Estero, entre 1928 y 1951. La colección contiene más de 4.000 objetos, piezas cerámicas en su mayoría, incluyendo también artefactos líticos y restos humanos.

Este trabajo es el resultado de un estudio de la colección von Hauenschild y del plan de manejo de colecciones que el Museo de Antropología de la UNC lleva a cabo para revalorizar sus colecciones en el depósito. Retomando los planteos de las perspectivas del manejo de colecciones en museos y a través del análisis del archivo personal de von Hauenschild, nos acercamos a comprender la trayectoria de un hombre que, desde la perspectiva actual, se inició como «huaquero» para luego convertirse en un arqueólogo autodidacta. El trabajo también explora el uso de métodos, innovadores para su época, que von Hauenschild aplicó en sus investigaciones arqueológicas de la provincia en la que residió por más de 30 años.

Palabras clave: Historia de colecciones, Jorge von Hauenschild, Santiago del Estero, Arqueología, Museo de Antropología

In the shadow of Argentinean archaeology: Jorge von Hauenschild and the formation of the von Hauenschild collection at the Museo de Antropología (Universidad Nacional de Córdoba)

\section{Abstract}

This is a study of the von Hauenschild collection located at the Museo de Antropología, Facultad de Filosofía y Humanidades of the Universidad Nacional de Córdoba, Argentina. The study is an exploration of the history of the collection through time and space and how it was formed and collected in the province of Santiago del Estero by the German born engineer, Jorge von Hauenschild between 1928 through 1951. The collection consists of over 4000 objects, the largest amount of elements in the collection is ceramics, but it also includes various lithic artifacts and human remains.

This paper is the result of the study of the von Hauenschild collection as a part of the Museo de Antropología, (UNC) plan of collection management which intends to revalorize it. From the perspective of collection management the objects in the collection is studied and through analysis of von Hauenschild's personal archive; we step closer to understand the trajectory of a man that started out as an advocate, in our present perspective, to later transform into a self-thought archaeologist. The study also tries to demonstrate that von Hauenschild used modern methods, for the time, to investigate and reveal the native heritage of the province where he lived for over 30 years.

Keywords: History of collections, Jorge von Hauenschild, Santiago del Estero, Archaeology, Museo de Antropología

\section{Introducción}

Una persona olvidada en la historia de la Arqueología argentina, es el alemán Jorge von Hauenschild, quien se radicó en el país en 1908 y realizó excavaciones en la provincia de Santiago del Estero desde fines de la década de 1920 hasta su muerte en 1951. Durante sus últimos años trabajó en el Instituto de Arqueología, Lingüística y Folklore «Dr. Pablo Cabrera» de la UNC (en 
adelante IALF) como ayudante investigador bajo la dirección de Antonio Serrano. El estudio propuesto aquí se basa en el archivo personal de Jorge von Hauenschild (en adelante APH) parte del actual Archivo del Museo de Antropología de la UNC.

\section{Contexto histórico}

La bibliografía sobre la Arqueología de la Provincia de Santiago del Estero es realmente escasa en comparación con otras provincias del Noroeste argentino.

La primera generación de arqueólogos en Argentina, como Francisco P. Moreno, Samuel Lafone Quevedo, Adán Quiroga y Juan B. Ambrosetti, se interesaron en algún momento por la arqueología santiagueña, no obstante no se registra la misma atención que la que se dio al área valliserrana. Quienes sí mostraron un interés sostenido en el área santiagueña fueron Emilio Roger Wagner y Duncan Ladislao Wagner, conocidos como «los hermanos Wagner» (Martínez et al. 2003), quienes difundieron sus ideas acerca de los orígenes de la «Civilización ChacoSantiagueña» y sus vínculos con el Viejo Mundo (Wagner y Wagner 1934). Las particularidades ligadas a este vínculo sugerido por los Wagner han sido analizadas por Martínez et al. (2003).

La Arqueología de fines del siglo XIX y principios del $X X$ tenía por objeto la recuperación de material guiada por cuestiones más bien estéticas ya que no existía la concepción de contexto arqueológico como se entiende e interpreta hoy.

La práctica de adquirir objetos para las colecciones era en muchos casos guiada por el fin de completar gabinetes de curiosidades, lugares donde objetos extraños y extraordinarios que eran presentados al público, otorgando al coleccionista un particular status social. En esos tiempos, no existían prácticamente las excavaciones estratigráficas, sino que la norma era la exhumación de objetos considerados de valor y la mayoría del material arqueológico, como huesos de humanos y animales y fragmentos cerámicos, entre otros, no eran estimables y por tanto descartados.

Después de que los hermanos Wagner abrieran los ojos al mundo acerca de lo que ellos definieron como «Civilización Chaco - Santiagueña», varios investigadores y aficionados de la época comienzan a interesarse por los materiales arqueológicos de la región. Entre ellos, von Hauenschild, quien años más tarde comienza sus exploraciones.

Del APH se desprenden actividades de otros investigadores en la región además de von Hauenschild. Henry Reichen de la Universidad de Friburgo en Suiza, llega a Santiago del Estero en 1938 (APH - documentos: 107,108, 109, 111 , 114). En 1931, el arquitecto Héctor Greslebin', profesor de Arqueología en el Instituto Nacional del Profesorado Secundario, fue a Santiago del Estero a estudiar su prehistoria visitando el museo Wagner y varias colecciones privadas, entre ellas la de Jorge von Hauenschild, Rafael Delgado y Jorge Argañaraz (APH - documento: 50). Héctor Greslebin también realizó excavaciones en la localidad de Beltrán (El Indio, 15 de Febrero 1941, APH - documento: 118 ).

\section{Biografía de Jorge von Hauenschild (1877-} 1951) - Contexto socio-histórico

\begin{abstract}
...»writing always transforms. The process of writing the past is in the present needs to become part of that which is to be understood in archaeology. The ultimate aim of much contemporary archaeological discourse is to put an end to writing, to get the story right. Empiricism inexorably encourages such a futile goal. To the contrary, there will be no correct stories of the past that are not themselves a product of a politics of truth. There can only be better or worse re-presentations of history: his [sic] story.» (Christopher Tilley 1994:73).
\end{abstract}

La mayoría de los documentos que encontramos en el archivo personal de von Hauenschild son cartas recibidas por él, sin embargo están ausentes las cartas enviadas a lo largo de su vida. Existe un «copiador de notas» para 1920-21, aunque en mal estado y la letra manuscrita es de lectura dificultosa. La mayoría de la correspondencia está en español, aunque algunas cartas han sido escritas en alemán, inglés y unas pocas en francés.

El primer documento, ordenado según criterio cronológico, encontrado en APH es un recorte del diario La Prensa de Febrero de 1913 informando que von Hauenschild junto con dos ingenieros (Echeverría y Rhodius) fueron seleccionados para realizar una evaluación de puentes en la Provincia de Corrientes (APH - documento: 1).

Durante los primeros años de la década de 1920, von Hauenschild trabajó en diversas obras públicas en el sur de Argentina. No hay mucha documentación relativa a esa época de su vida. Se comienza a encontrar más material a partir de su instalación en Santiago del Estero. La siguiente reseña se basa en varios documentos de su archivo personal y un documento biográfico encontrado en su archivo que da una buena descripción de su vida. Este texto (APH - documento: 329, 330, 331, 332) escrito en alemán y en español, parece haber sido redactado, probablemente por su esposa, después de la muerte de von Hauenschild. El estilo del documento se acerca a un obituario.

Jorge von Hauenschild, originalmente Georg nació en Tscheidt, Oberschlesien, Alemania, el 20 
de Mayo de 1877, hijo de Max von Hauenschild (Gutsbesitzer und Landrats im Kreise Cosel).

Destinado a una carrera militar, entró a los 15 años en la escuela de cadetes Walstatt, pasando luego a Lichterfelde en Berlin. En 1895 obtuvo el grado de teniente, en 1903, abandona la carrera militar y comienza a estudiar ingeniería en Dresden durante los siguientes cinco años.

En Junio de 1908, arriba a la Argentina, obtiene su primer empleo como ingeniero del Ferrocarril al Pacífico en Buenos Aires. En Mayo de 1909, se casa en Mendoza con Paula (Paulina) Runge (18881972), hija de Don Alberto Runge. No tuvieron hijos, y von Hauenschild no tenía familia en Argentina. Paula tenía una hermana, Berta Regnat, además de su padre en Argentina (APH - documento: 370). Berta tuvo por lo menos una hija, Emma Weigelt que más tarde participará en la historia de la colección. Von Hauenschild se quedó en Mendoza hasta fines de 1910, momento en que parte a Río Negro. Allí obtuvo tierras "vírgenes e inhóspitas convertidas en nuevas viviendas» (APH documento: 330). En 1911, se le otorga la ciudadanía argentina. Durante la primera guerra mundial ocupó puestos públicos en Río Negro y Neuquén.

En 1920, se emplea en el Departamento Nacional de Higiene en una campaña contra el Paludismo en Santiago del Estero. Trabajó en los alrededores de la ciudad capital en distintas obras de saneamiento. Se mudó a la ciudad de La Banda en esa misma provincia. En 1925, comienza con un contrato en la Dirección General de Irrigación para realizar un estudio de aprovechamiento de las aguas del Río Dulce.

Hasta 1926, trabajó en varias obras de irrigación y defensa del Río Dulce. La mayor parte de la correspondencia entre la década del 20 y primera parte el 30 , son cartas, facturas y diferentes estudios médicos realizados a von Hauenschild.

En 1926, obtuvo un nuevo empleo como profesor en la Escuela Industrial de la Nación de Santiago del Estero, trabajo que conservó hasta 1948, cuando se traslada a Córdoba a trabajar al IALF.

El comienzo de las investigaciones arqueológicas de von Hauenschild en Santiago del Estero

De acuerdo a una entrevista publicada en La Gaceta (San Miguel de Tucumán) el 27 de Octubre de 1934 (APH - documento: 78, 79) von Hauenschild, comienza a interesarse por la prehistoria de Santiago del Estero en 1925. Luego de que los hermanos Wagner presentaron su idea de que los restos cerámicos allí encontrados eran el documento de una civilización antigua y desconocida fue que comenzó a realizar excavaciones en la margen izquierda del Río Dulce.

Según otro artículo escrito por él mismo (La Prensa 7 de Abril 1935, APH - documento: 82, 83), comenzó sus investigaciones independientemente de los Wagner en 1928. Las primeras exploraciones fueron en un paraje llamado Quiroga, a tres leguas al norte de La Banda, siguiendo el trayecto del Río Dulce hacia el norte hasta Chaupi Pozo. Al sur de Quiroga encontró un extenso asentamiento arqueológico, cerca del sistema de riego La Cuarteada. Descubrió así, trazas de dos importantes pueblos, el mayor se encontraba en Quiroga, y el otro frente a Chaupi Pozo, y pequeños sitios entre ambos (APH - documento: 78, 79).

Según otro documento (APH- Documento 82) «en una zona ubicada a aproximadamente unos 200 $\mathrm{Km}$. de los asentamientos del Chaco» siguiendo la margen izquierda del Río Dulce. Un área de $50 \mathrm{Km}$. fue explorada en dirección SE - NW. Allí encontró varios indicadores sobre la ocupación prehistórica del área, a una distancia a $15 \mathrm{Km}$. del actual curso del río. Varios de los asentamientos fueron encontrados cerca del lugar donde la topografía revelaba un paleocauce. Encontró evidencia de un gran asentamiento de más de 100 hectáreas, con organización perfecta, calles delimitadas y orientado a los cuatro puntos cardinales (APH - documento 82).

Más tarde, von Hauenschild localizó varios sitios. Trabajó en Acosta, Quiroga, Chaupi Pozo, Bocatoma, La Cuarteada, Vilmer, Soria y Bajadita. Esto redundó en las grandes colecciones radicadas hoy en el Museo de Antropología (UNC). La mayoría de estos objetos pueden relacionarse con el sitio arqueológico de donde se extrajo pues cuentan con datos de procedencia.

Von Hauenschild, no sólo se interesaba en hallazgos arqueológicos, también exhumó varios especimenes paleontológicos, por ejemplo en Quiroga, excavó dos «defensas» de mastodonte

\section{Museo privado von Hauenschild}

Von Hauenschild montó un museo privado en su casa de La Banda, Ilamado Museo Arqueológico y Paleontológico del Ing. Hauenschild, localizado en la primera cuadra de la Calle España.

En 1934 la colección estaba constituida por más de 2000 piezas, en su mayoría cerámica y lítico (La Gaceta 27 de Octubre 1934, APH - documento: 78, 79) a las que se sumaban 100 hallazgos paleontológicos. En 1948, al mudar a Córdoba la colección, esta había crecido a más de 4000 objetos.

Según otro periódico de la época (Mundo Argentino 24 de Noviembre 1937 APH - documento: 99) la mayor parte de los visitantes eran escolares $y$ turistas.

\section{La colección von Hauenschild «de gira»}

Von Hauenschild, prestó objetos de su colección en varias oportunidades a museos y exposiciones. En 1934 varios objetos arqueológicos, tanto de su colección como la de los Wagner, fueron exhibidas en el Pabellón Santiago del Estero de la Feria Industrial Argentina 
realizada en Buenos Aires (APH documento 64, 67). Este evento fue registrado en varios periódicos entre ellos La Nación, Mundo, La Prensa (3 y 4 de Febrero 1934). Una foto de parte de la colección von Hauenschild, de urnas funerarias, se encuentra en La Prensa de la Feria Industrial. (12 de Abril 1934, APH documento 67). Von Hauenschild envió 272 artefactos en total a la Feria (La Gaceta 27 de Octubre 1934, APH documento 79).

Al abrirse la nueva sala en el Museo Municipal de Bellas Artes dedicada a la Arqueología y Paleontología, en Agosto de 1939, casi todos los objetos exhibidos provenían de la colección von Hauenschild (La Prensa Septiembre 1939 y El Mundo 14 de Agosto 1939, APH documento 99)

\section{El comienzo de la búsqueda de un nuevo hogar} para la colección

En 1942, von Hauenschild comienza a escribir a varios institutos universitarios de Antropología / Arqueología / Etnología del país, con el fin de encontrar un nuevo lugar donde albergar su colección, así como un lugar de trabajo para él, de modo de institucionalizar sus actividades ligadas al campo de la Arqueología (APH documento 123, 124, 125). Su propósito respecto de la colección era la venta.

Se contactó con el Instituto Miguel Lillo en la Universidad Nacional de Tucumán, la más prestigiosa Universidad del Norte Argentino para la época. De allí lo derivaron al Instituto de Antropología de la misma Universidad.

Si existieron razones para vender su colección, estas no son realmente claras, posiblemente tuvo algunos problemas financieros, o tal vez su idea fuera que la colección termine en un instituto universitario.

La Universidad Nacional de Tucumán (UNT) mostró un gran interés por la colección, y comenzaron las tratativas para la compra. Sin embargo esto nunca se concretó y entre tanto cambiaron las autoridades -el Rector y la administración- de la UNT.

Durante varios años, von Hauenschild intentó obtener un cargo en la UNT. Finalmente en Agosto de 1946, ingresa como «Organizador de la Sección Arqueología, Auxiliar de Segundo grado» en el Instituto de Antropología, Facultad de Filosofía y Letras, de la UNT con un salario de 300 pesos mensuales (APH documento 195, 196, 197, 198, 199).

Allí trabajó junto a Antonio Serrano, quien en esa época tenía licencia de su cargo en Córdoba para trabajar en Tucumán como colaborador en la reorganización del Instituto de Antropología de la UNT. En Diciembre del mismo año, von Hauenschild deja su cargo en la UNT (APH documento 203, 204). De acuerdo a un documento encontrado sin fecha (APH documento 271) lo abandona debido a varias razones. Terminó de organizar las colecciones de la Sección de Arqueología y mucho de lo que quedó eran pequeños fragmentos y material sin procedencia, con los que no pudo hacer nada. En su decisión influyeron también la falta de espacio en el depósito, escasa carga horaria para su trabajo y falta de tiempo personal. Estas son las razones en el documento oficial, si hubo otros inconvenientes, no hay registro de ellos.

El siguiente año (1947) von Hauenschild estuvo en contacto con Serrano, aunque esta vez en Córdoba para obtener un cargo en este lugar. Esta vez la resolución se aprueba pero no pudieron ofrecer una suma suficiente de dinero para la compra de la colección.

Von Hauenschild obtiene el cargo en Córdoba, aunque tarda varios meses antes de mudarse definitivamente desde Santiago del Estero, lugar donde mantuvo su propiedad. Serrano le escribió varias veces reclamando su presencia en Córdoba para tomar posesión de su cargo. No obstante esto, von Hauenschild, continuaba en contacto con la UNT, puesto que su objetivo era obtener un cargo estable (diferente al que había tenido) en esa Universidad. En Diciembre de 1947, Osvaldo L. Paulotti, del Instituto de Antropología de la UNT, sugiere a von Hauenschild, aceptar su cargo en Córdoba hasta tanto lograra acceder a un puesto en Tucumán (APH documento 234). Paulotti cuenta a von Hauenschild en la misma carta, que el presupuesto de la Universidad aun no estaba aprobado, aunque existía la idea de contratar profesores del exterior. Esto fue en 1947, luego de la Segunda Guerra Mundial. En Marzo de 1948, Paulotti, vuelve a escribir informándole de un puesto como profesor adjunto de Prehistoria y Arqueología con un salario de $\$ 800$ mensuales (APH documento 243). Nunca se materializaron estas promesas y von Hauenschild se queda trabajando en Córdoba hasta su muerte en 1951.

von Hauenschild y el Instituto de Arqueología, Lingüistica y Folklore «Dr. Pablo Cabrera», UNC

El primer contacto con el IALF aparece en una carta enviada en 1943, en la cual se le informa que Antonio Serrano recibió dos publicaciones suyas (von Hauenschild 1943a; 1943b). Von Hauenschild las había enviado desde su casa en La Banda (APH documento 126). Más tarde, Serrano vuelve a escribir ofreciéndole publicar un artículo en la serie «Publicaciones» del IALF, sobre la Arqueología de Santiago del Estero. Serrano le deja «manos libres» en cuanto a la extensión del artículo y ofrece costear los gastos de fotografías e ilustraciones (APH documento 128). Serrano estaba interesado en obtener algunos objetos de Santiago del Estero para la colección del IALF de Córdoba. Von Hauenschild escribe (APH documento 129) que el puede arreglar esa cuestión si Serrano viaja a Santiago del Estero donde 
fácilmente podrá obtener objetos arqueológicos. Esto nos da una idea de cómo el patrimonio arqueológico era tratado, como algo de fácil compra-venta, un modo muy ligado al coleccionismo. El objetivo de Serrano era obtener colecciones de todo el país para su instituto, particularmente del centro de la Argentina. Su idea era crear el mejor centro de investigaciones del centro del país, lo cual expresa en su carta a von Hauenschild.

Ese mismo año (1943) se estableció un contacto epistolar regular entre von Hauenschild y Serrano, hasta el traslado a Córdoba por parte de von Hauenschild. Serrano le hablaba no sólo de obtener material para el IALF, también de viajes de campo que planificaba a Santiago del Estero. Le solicita a von Hauenschild que medie con Emilio Wagner, director del Museo en Santiago del Estero, a fin de poder visitar y examinar las colecciones en esa provincia.

Ese mismo año, 1943, von Hauenschild escribe a Serrano informándole que había trabado amistad con Duncan Wagner, aunque no tenía ningún tipo de relación con su hermano Emilio (APH documento 129).

En 1944, Serrano establece el primer contacto con el Rector de la UNC con el fin de lograr un cargo para von Hauenschild y comprar su colección (APH documento 143, 144, 145). Serrano brega por esto hasta 1947, cuando finalmente la Universidad se decide a comprar la colección y además obtiene una nominación como Ayudante Investigador del Instituto de Arqueología, Lingüística y Folklore para von Hauenschild (APH documento 227; Ferreyra 2006:126). Este es pagado con un salario mensual de 350 pesos.

Serrano pide a la UNC 10.000 pesos para comprar la colección, suma muy difícil de obtener. El pago finalmente se realiza en 1948 después de una larga negociación con el sistema universitario. Hay que considerar el valor del monto solicitado en comparación a los salarios de la época. Serrano urge a von Hauenschild para que se instale en Córdoba y tome posesión de su cargo en Enero o Febrero de 1948, luego de varios meses, von Hauenschild se instala en Córdoba. El mayor problema en el momento era el pago de la colección.

Von Hauenschild no deja su cargo en la Escuela Industrial de la Nación, en Santiago del Estero, sólo pide una licencia. En Noviembre 1948 las autoridades de esta escuela le escriben pidiendo que por favor vuelva a dar clases como corresponde a su cargo después de un año en Córdoba.

En 1949, mientras se desempeña en el IALF, von Hauenschild realiza un viaje de campo a las Sierras de Ojo de Agua y Sumampa en Santiago del Estero (APH documento 290, 291), existe un informe de ese viaje.
Trabajó en el IALF hasta su muerte en 1951. La correspondencia en los últimos meses de su archivo temporal, evidencian lo inestable de su estado de salud. Falleció el 2 de Noviembre de 1951, fue sepultado el sábado 3 de Noviembre de ese año en el cementerio de Villa Carlos Paz, localidad donde vivía con su esposa Paula (APH documento 320). Hoy, Jorge se encuentra sepultado junto a su esposa Paula en una tumba en cementerio de Villa Carlos Paz, en una fosa ( $\mathrm{N}^{\circ} 31$, Tablón 7 sección 1).

\section{Contactos internacionales}

Von Hauenschild estableció durante su vida numerosos contactos con antropólogos y arqueólogos de diversos países, como por ejemplo Colombia, EEUU, Suecia, Alemania y Suiza. Tenía también una extensa red ligada a investigadores en el mismo campo en Argentina y Chile. En Argentina, se destacan entre los más conocidos, Salvador Canals Frau, Antonio Serrano, José Imbelloni y Alberto Rex González; en Chile Grete Mostny; en Suecia Gerhard Lindblom, Sigvald Linné, Stig Rydén y Magnus Mörner; en Alemania, Karin Hissink y Hermann Trimborn.

Se relacionó con investigadores que trabajaban en Colombia y estuvo muy interesado respecto de un hallazgo arqueológico del valle del Río Ranchería. El recorte del diario está en su archivo (La Prensa 1 de Marzo de 1941, APH documento 119) junto a una carta escrita sobre el tema un par de días más tarde al Dr. Víctor Oppenheim (APH documento 120).

Von Hauenschild también estaba en contacto con museos en Estados Unidos. En 1936, recibe una carta del Museum of the American Indian, Heye Foundation de Nueva York, luego de haber publicado un artículo en el diario La Prensa (7 de Abril de 1935). Ellos se interesaron en saber si von Hauenschild tenía más publicaciones sobre las cerámicas del Chaco-Santiago. En realidad estaban interesados en obtener piezas para su museo. En la carta (APH documento 91) se le consulta gentilmente si estaba interesado en realizar un intercambio de piezas duplicadas entre ambas partes. De estar interesado debía informarle que especimenes y objetos de que áreas pretendía recibir $^{2}$.

Los intercambios eran una práctica usual entre los museos de diversos lugares durante gran parte del siglo $X X^{3}$. En algunos museos esta costumbre aún persiste. Los directores de museos solían donar piezas cuando viajaban y visitaban otros museos fuera de sus países. Afortunadamente, esta práctica ha sido abolida actualmente en los museos, hoy más profesionalizada, provista por el Código de deontología del ICOM.

Durante el Proceso Militar en Argentina, se observó también la práctica por parte de los interventores de regalar objetos de colecciones de museos públicos a particulares. Ese fue el caso de gran parte de la colección de Folklore, 
particularmente instrumentos musicales, que desaparecieron del Instituto de Antropología de la UNC (D. Quiroga com.pers., Marzo 2008).

\section{Contactos con los museos etnográficos de Suecia}

Los investigadores suecos tuvieron un temprano interés en el continente sudamericano. Erland Nordenskiöld, director del Departamento Etnográfico del Museo de Göteborg ${ }^{4}$ fue un pionero en realizar trabajos en el noroeste de Argentina, región chaqueña y Bolivia, transformándose en una verdadera influencia para futuros investigadores (Lindberg 1996). Sus discípulos suecos del Museo Etnográfico continuaron trabajando en el área generando extensas colecciones de referencia de todo el continente sudamericano.

Stig Rydén, discípulo de Nordenskiöld, se convirtió más tarde en curador del Museo Etnográfico. Trabajó en Candelaria (Salta) y Tiwanaku en Bolivia. Rydén y von Hauenschild se escribían seguido. En 1947 von Hauenschild envió muestras cerámicas a Gotemburgo.

El 22 de Mayo de 1947 W.A. Ruysch van Gorkum escribió a von Hauenschild (APH documento 208) desde su cargo en ETHNOSarchivo de Etnologia, Antropología y Arqueología. Ruysch cuenta a von Hauenschild que finalmente irá a Santiago del Estero a visitarlo el 8 o 9 de Junio, y que lo acompañaría el Conde Magnus Mörner ${ }^{5}$. Según su carta, Ruysch también contactó a Orestes di Lullo para dar una conferencia. Mörner, por su parte daría una conferencia acerca de las relaciones entre Suecia y los estados sudamericanos durante el siglo XIX. A su vez, de parte de Stig Rydén, Ruysch solicita a von Hauenschild enviar algunas muestras cerámicas al Museo Etnográfico de Gotemburgo puesto que no disponía de material del área Chacosantiagueña. Mörner es quien envía las muestras (APH documento 218). Luego a través de la Legación Sueca en Buenos Aires, von Hauenschild recibe las publicaciones de Rydén como contraparte del intercambio (APH documento 224).

Ruysch, mas tarde envía material al Museo Etnográfico de Gotemburgo de otras regiones de la Argentina. La Colección llega en 1948 registrada bajo el número $1948.1^{6}$

Esta está constituida por 42 piezas arqueológicas de la Argentina, solo un objeto de Santiago del Estero, y el resto procedentes de Catamarca, La Rioja, Tucumán y Salta. El objeto de Santiago del Estero procede de Estación Fernández, lugar donde von Hauenschild había recuperado piezas anteriormente. En base a su procedencia, es posible que la pieza enviada a Suecia haya sido colectada por von Hauenschild. La colección fue cambiada por una publicación de Etnologiska Studier ${ }^{7}$ (Fasth 2003:35). Si la colección incluía material de von Hauenschild aun no fue determinado ${ }^{8}$.

Von Hauenschild mantuvo contacto con sus colegas suecos hasta su muerte, intercambiando correspondencia y publicaciones. Von Hauenschild estuvo también vinculado a Sigvald Linné y Gerhard Lindblom del Museo Etnográfico de Estocolmo.

\section{Donaciones a otros museos}

En Junio de 1937 el director del «Museo de Historia Natural de San Juan Cornelio Moyano», Carlos Rusconi, recibió una donación de restos paleontológicos de von Hauenschild, quien las había exhumado en Santiago del Estero. La colección estaba constituida por restos de Megaterio, Mastodonte y Toxodonte, y otras especies no determinadas.

Rusconi escribió a von Hauenschild agradeciendo su donación y solicitando además el envío de una urna con párvulo para exhibir en su museo (APH documento 95, 96). En esos tiempos esto era muy común, y la exhibición de restos humanos no era cuestionada y aun no lo es en muchos museos en diversos lugares.

\section{Miembro de asociaciones}

Von Hauenschild fue miembro de varias asociaciones científicas e intelectuales en Argentina. En Santiago del Estero fue miembro de Centro de Estudios Históricos. Allí confraternizó con varios intelectuales de la época como el Dr. Canal Feijóo y el Dr. Orestes Di Lullo (APH documento 73, El Liberal 1 de Agosto de 1934). En esas asociaciones dio algunas conferencias acera de la prehistoria de Santiago del Estero y la cerámica de ese periodo, temas de su mayor interés.

En Diciembre de 1934, von Hauenschild se convierte en miembro de la Academia Americana de la Historia (APH documento 81); en Septiembre de 1936, del Circulo de Altos Estudios en Rosario (APH documento 94). En Noviembre de 1937 se funda el Círculo de Representantes de la Prensa de La Banda y su primer presidente fue von Hauenschild (APH documento 101, 103).

En 1938 von Hauenschild fue elegido para formar parte de la Comisión Municipal Honoraria de Cultura de La Banda (APH documento 106, 1 13). Fue también miembro de la comisión de vecinos de La Banda (Crítica 17 de Julio de 1939, APH documento 116).

En Mayo de 1947 se convierte en socio activo de la Sociedad Argentina de Americanistas (APH documento 210). Von Hauenschild recibe una carta de Juan Delale, presidente de esa asociación invitándolo a participar en la reunión inaugural del primer encuentro de la sociedad, realizado el 2 de Junio de 1947 en el Instituto de Arte Americano 
dependiente de la Facultad de Arquitectura en Buenos Aires (APH documento 211).

\section{von Hauenschild en el campo}

von Hauenschild pasó gran parte de su tiempo en el campo estando a cargo de diversos proyectos de ingeniería hidráulica, lo cual hizo que conozca muy bien el terreno en Santiago del Estero. Durante sus campañas arqueológicas aprovechó ese conocimiento. Es muy probable que mientras trabajaba como ingeniero, los peones encontraran materiales arqueológicos. Esto debió atraer su atención por el tema. La variedad de obras públicas realizadas en Santiago (ferrocarril, obras hidráulicas, desmontes, etc.) hicieron que von Hauenschild considerara estos lugares ideales para investigar. Mucho material se recuperó cerca de paraderos ligados al ferrocarril, algunos de los sitios se encontraban al realizarse explotaciones industriales de arena y ripio y minas de grava (APH documento 122).

La descripción de los sitios (El Indio $n^{\circ} 7$ - 15 de Abril de 1941, APH documento 122) muestra que von Hauenschild en 1941 ya tenía un buen conocimiento de la Geología y la importancia de la estratigrafía para interpretar los objetos que hallaba. De hecho, realizaba perfiles geológicos en las proximidades de las áreas donde realizaba hallazgos. Hizo descripciones del mal estado de conservación de los restos humanos, mencionando que en ocasiones solo se encontraba un «polvillo gris» en la mayoría de los sitios.

Esto muestra un avance metodológico respecto de una carta enviada por Alfredo Castellano en 1934 (APH documento 75), donde se sugiere a von Hauenschild tomar fotografías de los hallazgos, mapear los sitios, hacer perfiles de los estratos y tomar muestras de estos.

La colección del Ing. von Hauenscild radicada en el Museo de Antropología de la Universidad Nacional de Córdoba

"Collections management is the term used for the body of practices and procedures which control the regulated acquisition and disposal, preservation and care, security and accountability, movement and loan and, finally but most importantly, documentoation of objects within an institution. It is a crucial area in the information structure of any museum. Collections management involves the administration of all the objects which make up a museum's collections. Precise information is constantly needed about what an object is, where it is and where it has been» (Gwyn Miles 1988:162-163).

La colección von Hauenschild está radicada actualmente en la Reserva Patrimonial del Museo de Antropología, UNC. Está compuesta por más de 4000 objetos, varios de estos se encuentran hoy exhibidos al público en salas del Museo (Lindskoug 2008).

Von Hauenschild, finalmente, vendió parte de la colección a esta Universidad en 1948, en la misma época en la que obtuvo su trabajo en el IALF. Otra parte de la colección, que incluía su archivo personal (correspondencia, mapas, fotos y dibujos) fue donada al Instituto de Antropología de la UNC en 1976 por Emma Weigelt, sobrina de la esposa de von Hauenschild, sumándose al material ya radicado en el Instituto.

\section{La llegada de la colección von Hauenschild en 1948}

El traslado de la colección desde el museo privado en La Banda hacia Córdoba, resulto complejo. Serrano escribe a von Hauenschild (APH documento 237) antes de que la colección sea trasladada a Córdoba, sugiriendo para evitar el costo del material de embalaje, llenar los camiones con paja.

La distancia entre Santiago del Estero y Córdoba es de alrededor de $450 \mathrm{~km}$, actualmente el viaje toma aproximadamente 6 horas, aunque en la época debió tomar mucho más que esto. Dado que la colección incluía casi 4000 piezas, el traslado debió realizarse en cuatro etapas según los documentos encontrados en el archivo ${ }^{9}$. El responsable del transporte fue Andrés Carlos Seeven. El costo de cada uno de los cuatro transportes fue de $\$ 500$ ( $\$ 2000$ en total), el traslado implicó un $20 \%$ extra al costo de compra de la colección por parte de la UNC. Los viajes para tal fin se realizaron en las fechas: 28 de Mayo, 4 de Junio, 25 de Junio y 2 de Julio de 1948. Durante la época que se realizaban los traslados, von Hauenschild se encontraba en viaje de campo en Santiago del Estero. Salió de Córdoba el 10 de Mayo, y el gasto en esa expedición fue de $\$ 300^{10}$.

De acuerdo a los documentos encontrados, en el traslado en camiones se empleó paja para proteger los objetos. No disponemos de fotos ni otros documentos que registren el transporte de la colección, ni sabemos si hubo daños o pérdidas en el envío. Es fácil imaginar que dadas las condiciones la posibilidad de que hayan existido daños es alta.

Una vez radicados en el IALF, tanto von Hauenschild como la colección, él comienza a estudiarla con mayor detalle y a restaurar algunas piezas.

Análisis requeridos y practicados por von Hauenschild sobre los objetos de su colección

Von Hauenschild utilizó métodos estratigráficos para excavar y envió varios artefactos a diversos especialistas del país para realizar análisis. Para la época, el empleaba técnicas 
novedosas para extraer información de sus hallazgos. Su colección de cráneos fue analizada por José Imbelloni (von Hauenschild 1949:49). También envió restos humanos para su estudio a Carlos Rusconi en Buenos Aires entre 1933 y 1934 (APH documento 68) incluyendo una flauta de hueso animal que luego fue clasificado como «guanaco» (von Hauenschild 1943b:132).

En 1946, von Hauenschild contacta a Amparo Tartaglia en Córdoba con el fin de determinar la procedencia de arcillas de cerámica arqueológica (APH documento $181,182,185,186,187$ ). A su vez envió material al Laboratorio Químico de la Dirección de Minas, Geología e Industria de Córdoba para informarse acerca de la composición química de cerámicas de su colección (APH documento 294). También envió cuentas de collar a Martín Doello Jurado para determinar su procedencia (von Hauenschild 1943b:132).

\section{La donación de 1976}

Una segunda parte de la colección, como mencionamos anteriormente, fue donada al Instituto de Antropología por la sobrina política de von Hauenschild -siglada H76- N-.

Esta donación fue recibida el 23 de Marzo de 1976 por el Lic. Carlos Alfredo Romero, director del Instituto de Antropología de la UNC. Emma Weigelt -hija de la hermana de Paula - lo hace en representación de la Sra. Paula de von Hauenschild (APH documento 381). Esta donación fue realizada varios años después de la muerte del matrimonio von Hauenschild, Paula fallece en 1972. Porqué la colección fue donada en 1976 no ha sido establecido.

Esta sección de la colección esta constituida básicamente por libros, documentos personales y materiales arqueológicos y etnográficos. Tres listas acompañan el inventario, una de ellas registra el material arqueológico y etnográfico y esta constituida por 69 ítems, no obstante por tratarse en ciertos casos de lotes, el número de objetos asciende aproximadamente a 200. La segunda lista de 23 páginas concierne al material bibliográfico, 351 libros donados. La tercera lista de «varios» es lo que constituye puntualmente el Archivo Personal de Hauenschild (actualmente APH sobre el que se basa este trabajo) constituido por 41 ítems archivados en carpetas. Se trata de manuscritos, cartas, fotos, recortes de periódicos, dibujos, traducciones realizadas por von Hauenschild, documentos, etc.

Esta donación fue recibida, un día antes del golpe militar del 24 de Marzo del 76, por C. A. Romero, quien continuó a cargo del Instituto de Antropología de la UNC. En Mayo del mismo año, Romero eliminó de la institución toda la bibliografía que pudiera ligarse al marxismo, siguiendo directivas de las nuevas autoridades de la Universidad (Ferreyra 2006: 169).
La institución, la colección y el coleccionista. El contexto en el que la colección fue formada

La colección von Hauenschild fue formada en una época en que el coleccionismo era indiscriminado en muchas partes del mundo. Las instituciones y museos coleccionaban para fortalecer su posición dominante en el mundo (Barringer y Flynn 1998), y el legado colonial está siempre presente en la manera en que las colecciones eran formadas dentro de un paradigma colonial. El prestigio político, económico y social está fuertemente relacionado con las colecciones recolectadas en los museos y el Museo de Antropología de la UNC no fue una excepción en su conformación (Bonnin 2008).

Antonio Serrano, el primer director quiso crear un centro de estudios de la prehistoria en el centro de la nación argentina y lo hizo. Con la incorporación de la colección von Hauenschild al IALF, él logró apropiarse de una parte importante de la prehistoria argentina que era minimizada por la elite intelectual de Buenos Aires.

En su mayoría, la colección consiste en cerámicas, ya que los objetos de este tipo de material son aquellos que mejor se preservan en el registro arqueológico junto con los objetos líticos. No obstante, la moda de la época era coleccionar «piezas maestras» cerámicas (master pieces), ya que ello otorgaba al coleccionista prestigio social y una posición destacada en la sociedad. Esto podría haber sido incluso más importante para von Hauenschild, que tenía ascendencia alemana, ya que así podía asegurar su posición en la sociedad argentina, aún cuando se trataba de una época en la que en el país existían actitudes pro-Europa y muchos inmigrantes europeos estaban haciendo su entrada. Sin embargo, los alemanes contaban con mala reputación luego de la Primera Guerra Mundial y aún peor luego de la Segunda en varias partes del mundo. Martínez et al. (2003) hacen mención a la animosidad hacia los alemanes por parte de los hermanos Wagner que eran franceses. Así, cuando Antonio Serrano le escribe al Rector de la Universidad para solicitar el establecimiento del cargo de von Hauenschild, le hace conocer que se trata de un descendiente alemán pero que es un hombre con valores democráticos. Cabe resaltar que en esta época una de las disciplinas, la antropología, se encontraba fuertemente conectada con el desarrollo del colonialismo europeo que necesitaba comprender, interpretar y dominar al «otro», en este caso, al «otro» americano.

En la Argentina, esta época se caracteriza por el establecimiento de la arqueología/antropología en las universidades, como en muchos otros países de América, encontrándose la primera de las disciplinas estrechamente relacionada con la 
segunda. En muchos casos, el Estado argentino invitó a investigadores europeos a las universidades con el deseo de que «europeizaran» el país de acuerdo a los estándares de la élite argentina. La inmigración europea al país fue inmensa en la primera parte del siglo XX y fue sostenida por el gobierno local. Todo ello era sumamente importante para la «nueva» nación, especialmente después de la negación de los derechos indígenas y el exterminio de la Conquista del Desierto (en la última parte del siglo XIX). En este contexto tomaron parte influyentes antropólogos, quienes comenzaron a formar colecciones para los museos universitarios del país.

Como coleccionista, von Hauenschild se mostraba interesado en la cerámica -la moda de la época-, algo que se encuentra reflejado en la selección de los objetos y artefactos que componen la colección (aunque como se dijo más arriba es verdad que también se trata de uno de los materiales que se hallan con mayor frecuencia en la provincia). Este interés puede ser visto también en sus publicaciones y en el material que envió para el análisis, siendo que la mayoría se trataba de muestras cerámicas.

En el contexto de ese momento, la Provincia de Santiago del Estero había sufrido varios desastres ecológicos productos de las actividades de las compañías inglesas: deforestación y venta de maderas que permitía obtener dinero fácil, convirtiendo esa provincia otrora verde y forestada, en un desierto polvoriento. El proyecto de ferrocarril, que dejó tantas ciudades fantasmas, y el proyecto hidráulico para el cambio del curso del Río Dulce, en el que von Hauenschild tomó parte activa como ingeniero, posiblemente lo motivaron a intentar contribuir al fortalecimiento de la imagen y orgullo de esta provincia pobre y olvidada del centro del país, en la que él vivió más de 30 años. Su interpretación de la prehistoria de Santiago del Estero apuntaba a revalorizar lo local en contraposición a la postura de los Wagner (ver Wagner y Wagner 1934) que entendían los desarrollos tecnológicos como exógenos. Estas ideas no son explícitas en los documentos, no obstante sumergirnos en la biografía de este personaje, permitió entrever la tensión entre su intervención profesional (ingeniero) y su interés en los objetos arqueológicos y el pasado indígena, que debió incidir en su forma de percibir esto último.

La colección von Hauenschild puede ser vista como una colección científica en tanto Hauenschild realizó esfuerzos para actualizarse con las últimas interpretaciones y métodos arqueológicos con el objetivo de crear una nueva y vívida imagen de la prehistoria ${ }^{11}$. La colección puede ser comparada con muchas otras privadas de la misma época, formadas bajo otros paradigmas y no con el interés de formar una colección científica para responder a preguntas en torno a la prehistoria santiagueña, tal y como era el objetivo de von Hauenschild. El coleccionista pudo haber comenzado como un simple huaquero, según nuestra percepción actual, pero terminó trabajando como un profesional usando los métodos arqueológicos más modernos para la época.

Córdoba, 3 de Septiembre 2008

\section{Agradecimientos}

Quiero agradecer a Bernarda Marconetto y Francisco Pazzarelli por la ayuda en la traducción del artículo. Mirta Bonnin y Adriana Muñoz han realizado valiosos comentarios del manuscrito. Darío Quiroga, en la Reserva Patrimonial del Museo de Antropología me ha acompañado y guiado durante todo el trabajo con la colección von Hauenschild. Constanza Taboada realizó valiosos aportes que contribuyeron al desarrollo del texto.

\section{Bibliografía}

Barringer, T.y T.Flynn. 1998. Colonialism and the Object: Empire, Material Culture and the Museum, Routledge, London and New York.

Bonnin, M. 2008. La conformación de colecciones en la construcción de las antropologías locales: El Instituto de Arqueología, Lingüística y Folklore de Córdoba (1941-48). Manuscrito en posesión del autor.

Fasth, N. 2003. «La Candelaria-Preservation and Conservation of an Archaeological museum Collection from North-Western Argentina at the Museum of World Culture, Sweden.» Department of Environmental Science and Conservation, Institute of Conservation, Göteborg University, Suecia. Tesis de Maestría inédita.

Ferreyra, C. A. 2006. Museo, Ciencia y Sociedad en la Córdoba moderna. El Museo Histórico Provincial y el Museo de Antropología: pensamiento y práctica. Publicaciones, Universidad Nacional de Córdoba.

von Hauenschild, J. 1943a. Revista de la Junta de Estudios Históricos de Santiago del Estero. Año I, $\mathrm{N}^{\circ} 1$. Santiago del Estero.

von Hauenschild, J. 1943b. Los Aborígenes de Santiago del Estero. En: Revista de la Junta de Estudios Históricos de Santiago del Estero, Año I, №2., pp: 116-137, Santiago del Estero.

von Hauenschild, J. 1949. Ensayo de clasificación de la documentación arqueológica de Santiago del Estero. Universidad Nacional de Córdoba.

International Council of Museums (ICOM) 2006, Código de deontología del ICOM para los museos. 
Lindberg, C. 1996. Erland Nordenskiöld-ett indianlif. Kungliga Vitterhets Akademins Serie: Svenska Lärde. Bokförlaget Natur \& Kultur, Stockholm.

Lindskoug, H. B. 2008. «Mysteries from the inside of the earth, heritage hidden away in the depths of a deposit- A study in collection management of the von Hauenschild collection at the Museo de Antropología, UNC, Argentina.» Museion, Göteborg University, Suecia. Tesis de Maestría inédita. 77 paginas.

Martínez, A. T., C. Taboada y L. A. Auat. 2003. Los Hermanos Wagner: entre ciencia, mito y poesía. Arqueología, campo arqueológico nacional y construcción de identidad en Santiago del Estero 1920-1940. Ediciones Universidad Católica de Santiago del Estero. Santiago del Estero.

Miles, G. 1988. Conservation and Collection Management: Integration or Isolation. The International Journal of Museum Management and Curatorship 7: 159-163.

Mörner, M. 1985. Actividades políticas y económicas de los jesuitas en el Río de la Plata. Editorial Hyspamérica.

Mörner, M.1953. The political and economic activities of the Jesuits in the La Plata region. The Hapsburg Era. Library and Institute of IberoAmerican Studies, Stockholm.

Ocampo, B. 2005. La Nación Interior-Canal Feijóo, Di Lullo y los Hermanos Wagner- El Discurso culturalista de estos intelectuales en la provincia de Santiago del Estero. Antropofagia, Buenos Aires.

Pearce, S. 1997. Archaeology as collection. En: Representing Archaeology in Museums, G.T. Denford (editor), The Museum Archaeologist 22: 6-12.

Tilley, C. 1994. Interpreting material culture. En: Interpreting Objects and Collections. Susan M. Pearce (editor). Leicester Readers in Museum Studies. Routledge, London and New York, pp: 67-75.
Wagner, E. y D. Wagner. 1934. La Civilización Chaco-Santiagueña y sus correlaciones con las del Viejo y Nuevo Mundo. Tomo I, Buenos Aires.

\section{Periódicos consultados \\ La Gaceta (San Miguel de Tucumán) \\ Mundo (Santiago del Estero) \\ La Nación (Buenos Aires) \\ La Prensa (Buenos Aires) \\ Critica (Buenos Aires) \\ El Indio (Buenos Aires) \\ El Liberal (Santiago del Estero) \\ Mundo Argentino}

\section{Comunicación Personal}

Jan Amnehäll, Responsable de las colecciones, Museo de Cultura Mundial, Gotemburgo, Suecia.

Adriana Muñoz, Curador, Museo de Cultura Mundial, Gotemburgo, Suecia.

Darío Quiroga, Responsable de conservación preventiva en el Museo de Antropología, UNC, Córdoba, Argentina.

' Parte de su colección fue donada al actual Museo de Antropología (UNC).

2 Si se realizó un intercambio, no hay información en el archivo (ver APH documento 91, 92, 93).

3 Durante esta época era común enviar muestras o pequeñas colecciones de referencia a otros museos alrededor del mundo. Serrano envió 25 objetos al Museo Etnográfico en Gotemburgo en 1930 de Paraná (Adriana Muñoz com.pers. Mayo 2008).

4 El Departamento Etnográfico del Museo de Gotemburgo, más tarde se transformó en un museo independiente en 1946, el Museo Etnográfico. En 1999 cambió nuevamente de nombre por el de Museo de Cultura Mundial (VKM en adelante) fue abierto al público en 2004.

${ }_{5}$ Magnus Mörner fue otro científico sueco interesado en la historia Latinoamericana. Se especializó en temas argentinos, en particular la expulsión de los Jesuitas de la Misiones. Tema sobre el cual escribió su tesis doctoral (Mörner 1953, 1985).

${ }^{6}$ Número de Colección de VKM

7 Etnologiska Studier era una publicación del Museo Etnográfico de Gotemburgo

${ }^{8}$ Según Jan Amnehäll del VKM no hay referencia a Jorge von Hauenschild en las colecciones de Museo (com. Pers. Abril 2008).

9 Encontrado en Plan de gastos 1948. Archivo del Museo de Antropología (AMA, UNC).

10 Plan de gastos 1948. Vale $n^{\circ} 5$. (AMA, UNC). 